\title{
Rice of Northeast India harbor rich genetic diversity as measured by SSR markers and $\mathrm{Zn} /$ Fe content
}

\author{
Vanlalsanga, S. Priyokumar Singh and Y. Tunginba Singh ${ }^{*}$ (ID
}

\begin{abstract}
Background: Rice (Oryza sativa L.) is one of the most important crops of the world and a major staple food for half of the World's human population. The Northeastern (NE) region of India lies in the Indo-Burma biodiversity hotspot and about $45 \%$ of the total flora of the country is found in the region. Local rice cultivars from different states of NE India were analyzed for genetic diversity and population structure using microsatellite markers, and their zinc and iron content.

Results: A total of 149 bands were detected using twenty-two microsatellite markers comprising both random and trait-linked markers, showing 100\% polymorphism and high value of expected heterozygosity (0.6311) and the polymorphism information content (0.5895). Nali Dhan cultivar of Arunachal Pradesh possessed the highest genetic diversity (0.3545) among studied populations while Moirangphou Khonganbi of Manipur exhibited the lowest genetic diversity (0.0343). The model-based population structure revealed that all the studied 65 rice cultivars were grouped into two clusters. Cluster I was represented by 36 cultivars and cluster II by 29 cultivars. Badalsali cultivar of Assam possessed the highest Zn content $(75.8 \mu \mathrm{g} / \mathrm{g})$ and Kapongla from Manipur possessed the lowest $(17.98 \mu \mathrm{g} /$ g). The highest and the lowest Fe content was found in Fazu $(215.62 \mu \mathrm{g} / \mathrm{g})$ and Idaw $(11.42 \mu \mathrm{g} / \mathrm{g})$ of Mizoram.
\end{abstract}

Conclusion: The result suggested rice cultivars of NE India possessing high genetic diversity (Nali dhan), high Zn (Badalsali) and Fe (Fazu) content can be useful as a source of germplasm for future rice improvement programs.

Keywords: Northeast India, Rice landraces, Genetic diversity, Microsatellite marker, Zn/ Fe content

\section{Background}

Rice (Oryza sativa L.) is the staple food for more than $50 \%$ of the world's population. The rice production and consumption in Asia alone accounts for more than $90 \%$ of the global rice yields [1]. It is imperative to develop measures to improve global rice production to warrant food security for increasing human populations. Although rice production has increased to about two folds in the past few decades with the introduction of improved varieties and proper crop management strategies, the need for high yielding, better varieties still remain unchanged. Bouis and Welch [2] suggested that increased rice productivity and the ability to deliver all the essential nutrients is crucial to meet both the energy needs and adequate nutritional health for the people in

\footnotetext{
* Correspondence: tunginba@mzu.edu.in

Department of Botany, Mizoram University, Aizawl, Mizoram, India
}

developing countries. Kennedy et al. [3] have reported that more than two billion people are affected with Iron $(\mathrm{Fe})$, Iodine $(\mathrm{I})$, Zinc $(\mathrm{Zn})$, and vitamin A deficiencies, especially in poor families of developing countries, of which more than five million children die every year due to nutrient malnutrition [2]. $\mathrm{Fe}$ and $\mathrm{Zn}$ are essential micronutrients for all forms of life due to their functional importance in cell development and gene expression $[4,5]$. Zn deficiency is known to be one of the most important malnutrition problems [6]. The effects of $\mathrm{Zn}$ deficiency include growth retardation, diarrhea, emotional disorders, reduction or absence of hormone secretion in male adolescents, rough skin, poor appetite, mental lethargy, delayed wound healing, weight loss, etc. [7]. Fe deficiency leads to blood loss, mal-absorption, chronic diseases, genetic disorders, etc. [8-11]. Increased $\mathrm{Zn}$ and Fe uptakes are required during crucial metabolic periods such as early human growth and pregnancy, so

(c) The Author(s). 2019 Open Access This article is distributed under the terms of the Creative Commons Attribution 4.0 International License (http://creativecommons.org/licenses/by/4.0/), which permits unrestricted use, distribution, and reproduction in any medium, provided you give appropriate credit to the original author(s) and the source, provide a link to the Creative Commons license, and indicate if changes were made. The Creative Commons Public Domain Dedication waiver (http://creativecommons.org/publicdomain/zero/1.0/) applies to the data made available in this article, unless otherwise stated. 
children and pregnant women are at higher risk of these nutrients deficiency $[6,12,13]$. It has been suggested by rice workers that the development of rice varieties with higher nutrient content may improve the nutritional health of people whose major diet is rice.

The Northeastern states of India, comprising Arunachal Pradesh, Assam, Manipur, Meghalaya, Mizoram, Nagaland, and Tripura, lies within the international boundaries of Bhutan and China in the north, Bangladesh in the southeast and Myanmar in the west. This region constitutes the Indo-Burma biodiversity hotspot [14] and is inhabited by various ethnic groups of people who speak different dialects and perform different cultural practices. The topography and biogeography of the region make this place a picturesque and also rich in biodiversity of flora and fauna. About $45 \%$ of the total flora of the country is found in the region [15]. This region harbors the richest genetic diversity reservoir for agri-horticultural crops. Rice cultivation provides the main source of food and employment for the people of this region as most of the population is involved in agriculture and allied activities. About $72 \%$ of the total cultivated area is under agricultural cultivation practices in upland, lowland, and water fed areas [15]. Although a large number of rice cultivars are available, most of the rice cultivated in the region are high yielding varieties (HYV) developed using modern genetic engineering tools. This trend implies a possible narrowing of the natural gene pool. However, it is also surprising to know that the many indigenous farmers of the hilly areas are still practicing their own landrace or cultivar cultivation that they inherit from their forefathers, which suit the local microclimate and adaptation. The cultural importance of the local landraces is also depicted by these people.

Knowledge on the extent of genetic variation and relationship among genotypes is necessary for developing more effective breeding and conservation programs [16], Understanding and utilizing the genetic diversity in crop plants is crucial for sustaining the increasing global and local food demands [17]. Assessment of the genetic diversity of local rice landraces or cultivars will provide a valuable source as it can be useful for crop improvement programs, Integrated Pest Management (IPM) measures and sustainable development of agriculture. Rice varieties of this region possess unique traits which are of great interest to the plant breeders. Some of the useful qualities identified in these landraces include unique adaptive traits for cold tolerance, flooding and salt tolerance, etc. [15]. Many molecular markers have been used to assess genetic diversity within and between populations. Among them, microsatellite or SSR (Simple sequence repeat) are one of the most preferred for assessment of genetic diversity because they are reliable, rapid, easy to score, cost-effective and require only a small amount of DNA $[18,19]$. The present study was performed to assess the genetic diversity of the local rice landraces of
Table $1 \mathrm{Zn}$ and Fe content, Gene diversity and percentage polymorphism in the studied cultivars

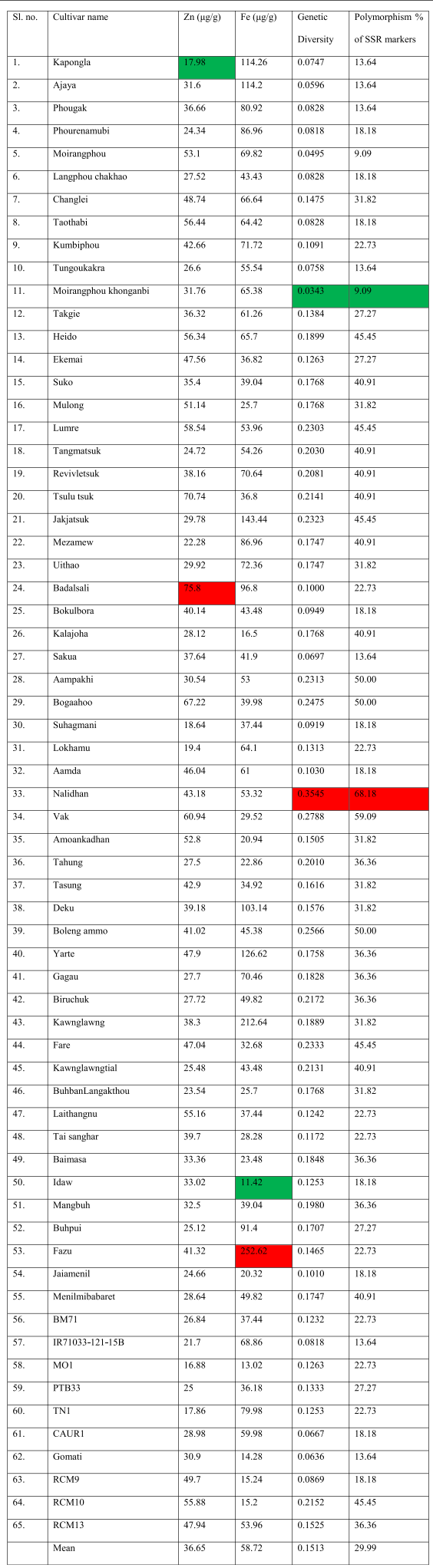

Highest value cell was indicated in red colour and lowest value cell was indicated in green colour for each parameter 


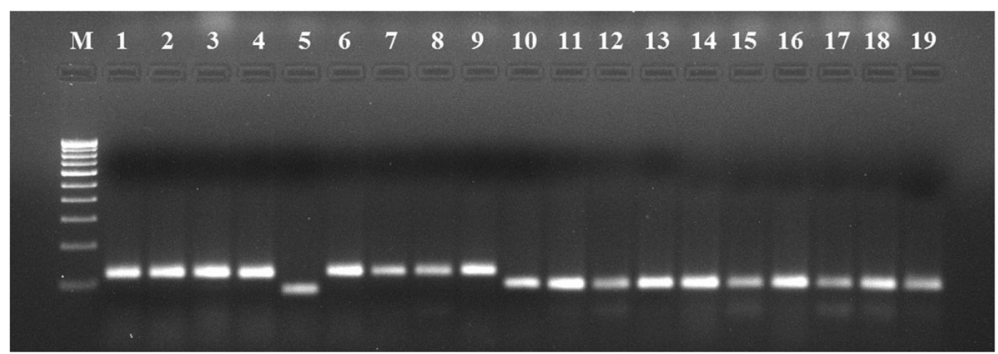

Fig. 1 A 2.5\% agarose gel showing the banding pattern of Assam rice cultivars generated by RM1. M represents a 100 bp DNA ladder. Lane 1-9 Suhagmani, 10-19 Lokhamu

the Northeast states of India using SSR markers, with two aims i) to estimate the $\mathrm{Zn}$ and $\mathrm{Fe}$ contents and ii) to facilitate conservation and utilization of these landraces.

\section{Results}

\section{$\mathrm{Zn}$ and Fe content}

Table 1 summarizes the $\mathrm{Zn}$ and Fe content of rice cultivars used in this study. $\mathrm{Zn}$ content in the studied cultivars ranged from $17.98 \mu \mathrm{g} / \mathrm{g}$ to $75.8 \mu \mathrm{g} / \mathrm{g}$ with an average of $36.65 \mu \mathrm{g} / \mathrm{g}$ (Table 1). Badalsali cultivar of Assam possessed the highest $\mathrm{Zn}$ content and Kapongla of Manipur possessed the lowest. The $\mathrm{Zn}$ contents of Northeast rice cultivars $(38.55 \mu \mathrm{g} / \mathrm{g})$ were higher than that of improved varieties $(32.17 \mu \mathrm{g} / \mathrm{g})$ used in the current investigation.

Fe content ranged from Idaw $(11.42 \mu \mathrm{g} / \mathrm{g})$ to Fazu $(215.62 \mu \mathrm{g} / \mathrm{g})$ with an average of $59.29 \mu \mathrm{g} / \mathrm{g}$ (Table 1). Similar to Zn, Fe contents of Northeast rice cultivars $(62.9 \mu \mathrm{g} / \mathrm{g})$ were higher than that of improved varieties $(39.41 \mu \mathrm{g} / \mathrm{g})$ in the present study.

\section{SSR polymorphism}

The agarose gels showing banding patterns of some rice cultivars were presented in Figs. 1 and 2. Table 2 shows a summary of the genetic markers used in the current study. A total of 149 bands were detected using twentytwo SSR primers. All twenty-two SSR markers were found to be polymorphic (100\% polymorphism). The average number of alleles per locus was 6.7727 and the maximum number of band [12] was generated by RM223 and the minimum [2] was generated by RM315. The mean number of effective alleles was found to be 3.3080. Major allele frequency (MAF) ranged from 0.2431 (RM246) to 0.9723 (RM443) with an average of 0.4973 . Expected heterozygosity $\left(\mathrm{H}_{\mathrm{E}}\right)$ varied from 0.1157 (RM315) to 0.8466 (RM72) with an average of 0.6311 respectively. Nei's genetic diversity varied from 0.1155 (RM315) to 0.8453 (RM72) with an average of 0.6301. Fst ranged from 0.5739 (RM223) to 0.9619 (RM443) with an average of 0.7870 . Polymorphism information content (PIC) ranged from 0.1012 (RM315) to 0.8274 (RM72) averaging 0.5895. The summary statistics of each marker is shown in Table 2.

\section{Population structure analysis}

The model-based population structure analysis using STRUCTURE showed that the highest value of $\Delta \mathrm{K}$ was at $\mathrm{K}=2$ (Fig. 3), grouping all the studied 65 rice cultivars into two clusters (Fig. 4), designated here as cluster I and cluster II. Principal Coordinates Analysis (Fig. 5) performed using GenAlEx and UPGMA tree (Fig. 6) constructed using MEGAfurther supplemented the STRUCT URE results. Both PCoA and UPGMA tree divided 65 rice

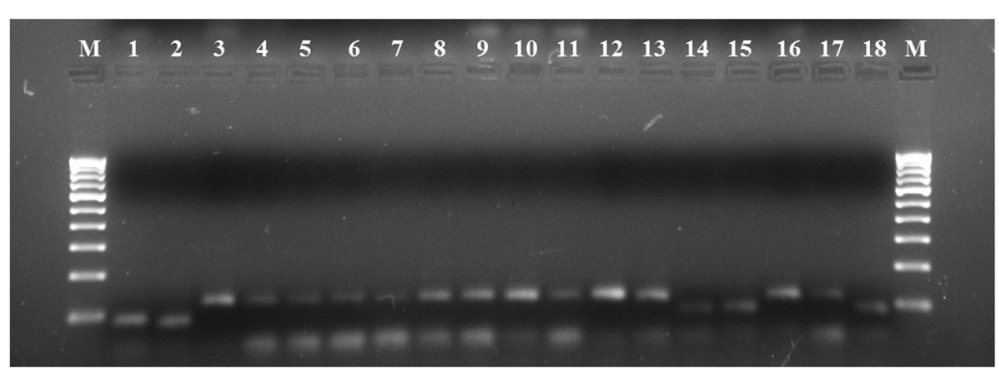

Fig. 2 A 2.5\% agarose gel showing the banding pattern of Nagaland rice cultivars generated by RM1. M represents a 100 bp DNA ladder. Lane 12 Heido, 3-12 Ekemai, 13-18 Suko 
Table 2 Summary of markers used in the present study

\begin{tabular}{|c|c|c|c|c|c|c|c|}
\hline Locus & na & ne & MAF & $\mathrm{H}_{\mathrm{E}}$ & Nei & Fst & PIC \\
\hline RM1 & 10.0000 & 4.2403 & 0.3662 & 0.7653 & 0.7642 & 0.8164 & 0.7359 \\
\hline RM154 & 8.0000 & 5.1918 & 0.3200 & 0.8086 & 0.8074 & 0.7367 & 0.7439 \\
\hline RM131 & 11.0000 & 2.5082 & 0.6123 & 0.6022 & 0.6013 & 0.5789 & 0.5826 \\
\hline RM135 & 9.0000 & 5.5756 & 0.2738 & 0.8219 & 0.8206 & 0.5358 & 0.7963 \\
\hline RM153 & 4.0000 & 1.9540 & 0.5815 & 0.4890 & 0.4882 & 0.9389 & 0.3726 \\
\hline RM125 & 5.0000 & 1.9837 & 0.6231 & 0.4967 & 0.4959 & 0.7735 & 0.4161 \\
\hline RM72 & 11.0000 & 6.4654 & 0.2569 & 0.8466 & 0.8453 & 0.7703 & 0.8274 \\
\hline RM171 & 5.0000 & 2.5417 & 0.5308 & 0.6075 & 0.6066 & 0.7768 & 0.5570 \\
\hline RM287 & 4.0000 & 2.7942 & 0.4846 & 0.6431 & 0.6421 & 0.7614 & 0.6151 \\
\hline RM302 & 5.0000 & 1.4456 & 0.8154 & 0.3087 & 0.3082 & 0.8473 & 0.2922 \\
\hline RM3825 & 4.0000 & 2.4690 & 0.5600 & 0.5959 & 0.5950 & 0.9255 & 0.5624 \\
\hline RM246 & 10.0000 & 5.6321 & 0.2431 & 0.8237 & 0.8224 & 0.7969 & 0.8036 \\
\hline RM260 & 6.0000 & 3.5529 & 0.3662 & 0.7196 & 0.7185 & 0.8784 & 0.6710 \\
\hline RM525 & 6.0000 & 2.8050 & 0.5431 & 0.6445 & 0.6435 & 0.9460 & 0.5986 \\
\hline RM219 & 5.0000 & 2.8788 & 0.4815 & 0.6536 & 0.6526 & 0.9071 & 0.5998 \\
\hline RM315 & 2.0000 & 1.1306 & 0.9292 & 0.1157 & 0.1155 & 0.8721 & 0.1012 \\
\hline RM223 & 12.0000 & 3.4552 & 0.4985 & 0.7117 & 0.7106 & 0.5739 & 0.7060 \\
\hline RM8094 & 8.0000 & 3.4626 & 0.3646 & 0.7123 & 0.7112 & 0.6881 & 0.6587 \\
\hline RM493 & 8.0000 & 3.9689 & 0.3431 & 0.7492 & 0.7480 & 0.8976 & 0.7055 \\
\hline RM3412 & 7.0000 & 3.9858 & 0.3231 & 0.7503 & 0.7490 & 0.9166 & 0.7209 \\
\hline RM443 & 3.0000 & 1.4768 & 0.9723 & 0.3234 & 0.3229 & 0.9619 & 0.2471 \\
\hline RM169 & 6.0000 & 3.2572 & 0.4523 & 0.6941 & 0.6930 & 0.6990 & 0.6552 \\
\hline Mean & 6.7727 & 3.3080 & 0.4973 & 0.6311 & 0.6301 & 0.7870 & 0.5895 \\
\hline$S D \pm$ & 2.8273 & 1.4428 & 0.2034 & 0.1863 & 0.1860 & 0.1256 & 0.1920 \\
\hline
\end{tabular}

na Number of alleles, ne Effective number of alleles, MAF Major allele frequency, $H_{E}$ Expected heterozygosity, Nei Nei's genetic distance, Fst Genetic differentiation, PIC Polymorphism information content

cultivars into two groups. Cluster I was represented by 36 cultivars and cluster II was represented by 29 cultivars. In UPGMA tree, Cluster I was subdivided into four groups exhibiting rice cultivars of Manipur, Assam, Arunachal Pradesh, and Japonica varieties. And cluster II could also be subdivided into four groups comprising rice cultivars of Mizoram, Nagaland, Meghalaya and Indica varieties. Analysis of molecular variance (AMOVA) showed that the genetic variation of two clusters of 65 rice cultivars was distributed into $73 \%$ among populations and $27 \%$ within populations. Average distances (expected heterozygosity) between individuals in the same cluster varied from Cluster I (0.5197) to cluster II (0.5686). Fst values of Cluster I and Cluster II were found to be 0.2635 and 0.2107 respectively with an average of 0.2371 . The mean alpha value was found to be 0.0663 .

Population-wise diversity comparison (Table 1) showed that Nalidhan cultivar of Arunachal Pradesh possessed the highest genetic diversity (0.3545) among studied populations while Moirangphoukhonganbi of
Manipur exhibited the lowest genetic diversity (0.0343). In state-wise comparison, the genetic diversity $\left(\mathrm{H}_{\mathrm{E}}\right)$ of Manipur rice cultivars ranged from 0.0343 (Moirangphoukhonganbi) to 0.1475 (Changlei), and that of Nagaland ranged from 0.1263 (Ekemai) to 0.2323 (Jakjatsuk), and Assam, from 0.0697 (Sakua) to 0.2475 (Bogaahoo), Arunachal Pradesh, from 0.1030 (Aamda) to 0.3545 (Nalidhan), Mizoram, from 0.1172 (Tai sanghar) to 0.2333 (Fare) and Meghalaya rice cultivars ranged from 0.1010 (Jaiamenil) to 0.1747 (Menilmibabaret). Rice cultivars of Arunachal Pradesh, in overall exhibited highest gene diversity $(0.2022)$, followed by Nagaland (0.1896), Mizoram (0.1746), Assam (0.1492), Meghalaya (0.1378) and then Manipur (0.0788). The average genetic diversity of all indigenous cultivars was found to be 0.1575 which was higher than that of indica (0.1180) and japonica $(0.1170)$ varieties.

Comparative analysis of gene diversity, $\mathrm{Zn}$ and Fe content showed that there was no significant correlation among all the three parameters. However, few cultivars 


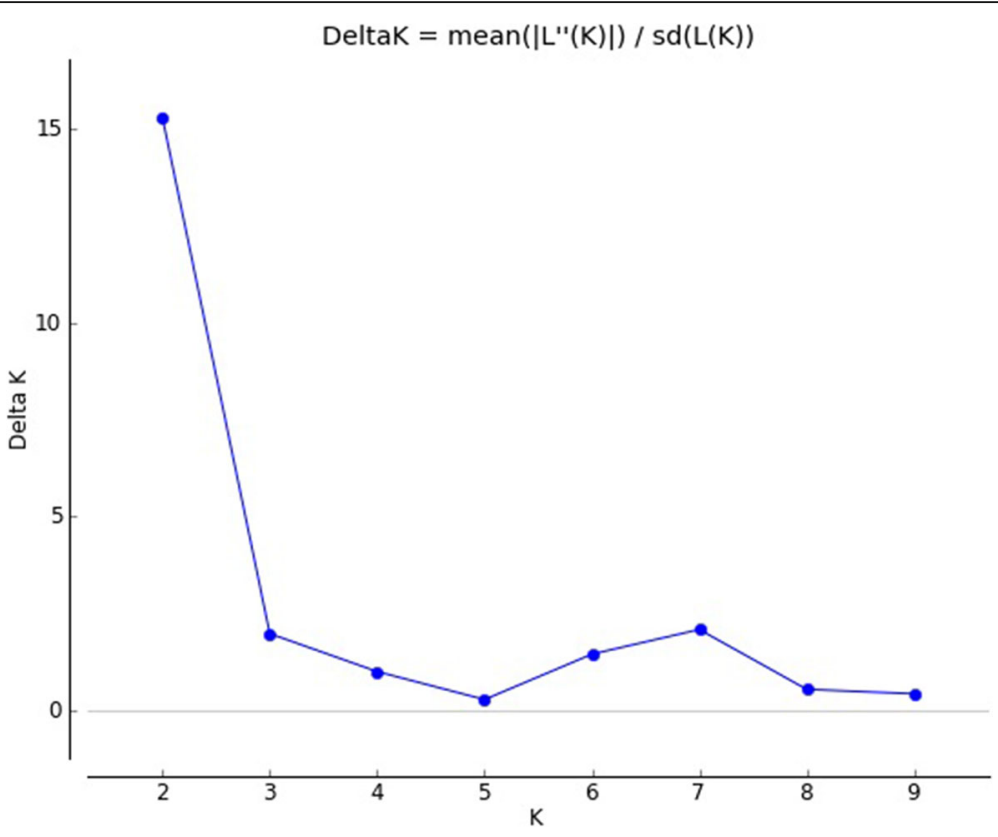

Fig. 3 Relationship between $\Delta K$ and $K$ showing a peak at $K=2$

with high gene diversity also showed higher $\mathrm{Zn}$ and Fe content (Table 3, Fig. 7).

\section{Discussion}

In this study, genetic structure and diversity analysis of 55 indigenous rice cultivars of Northeast India and 5 indica and 5 japonica test varieties were performed using twenty-two SSR markers comprising 9 random and 13 trait-linked markers, and $\mathrm{Zn}$ and $\mathrm{Fe}$ content. Assessments of genetic diversity of NE rice using molecular markers has been reported previously [20-25]. Though high genetic diversity was previously shown in the NE rice accessions, reports on micronutrients diversity are scarce. Micronutrient deficiencies to $\mathrm{Zn}$ and Fe, constitute the two most common nutrient deficiencies in humans $[23,26,27]$, especially in developing countries [28]. Although rice is a major staple food for a large part of the world especially in Asia, it has been reported as a poor source of essential micronutrients and vitamins
[29]. In the current study, relatively high Zn and Fe contents were detected in some of the cultivars. The Fe content in the present study was found to be higher than that of rice cultivars of West Bengal and adjoining areas, though zinc content was lower [23]. High Fe content was also previously reported in the Indian cultivars by Brar et al. [30]. The $\mathrm{Zn}$ content was higher and the $\mathrm{Fe}$ content was found to be lower than a previous report on local rice germplasm of Tripura state [31]. Average $\mathrm{Zn}$ and Fe contents in the present study were comparable with a previous report $[30,32]$. In another report by Verma and Srivastav [33], among some aromatic and nonaromatic Indian rice cultivars, aromatic rice had higher $\mathrm{Zn}$ and $\mathrm{Fe}$ contents. Interestingly, $\mathrm{Zn}$ and Fe contents in the current study was found to be higher than the ones reported by Verma and Srivastav [33]. Therefore, to overcome the micronutrient deficiencies, the present study will be helpful for designing crop improvement programs, though more investigations are still

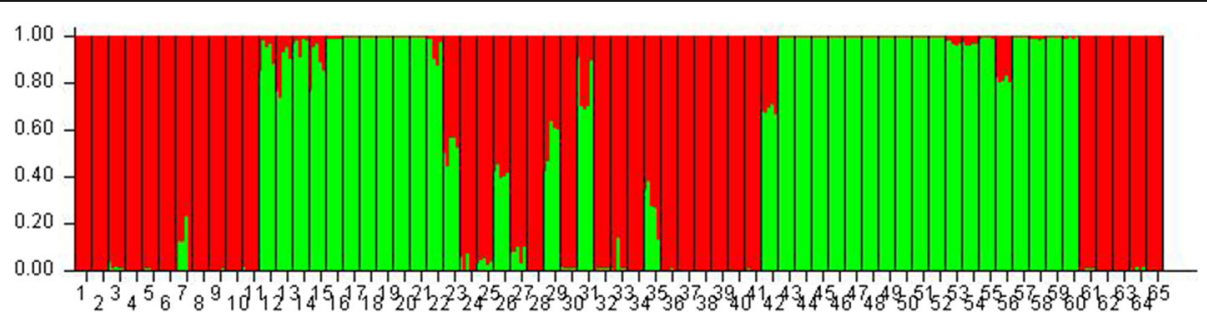

Fig. 4 Population structure (barplot) of rice cultivars 


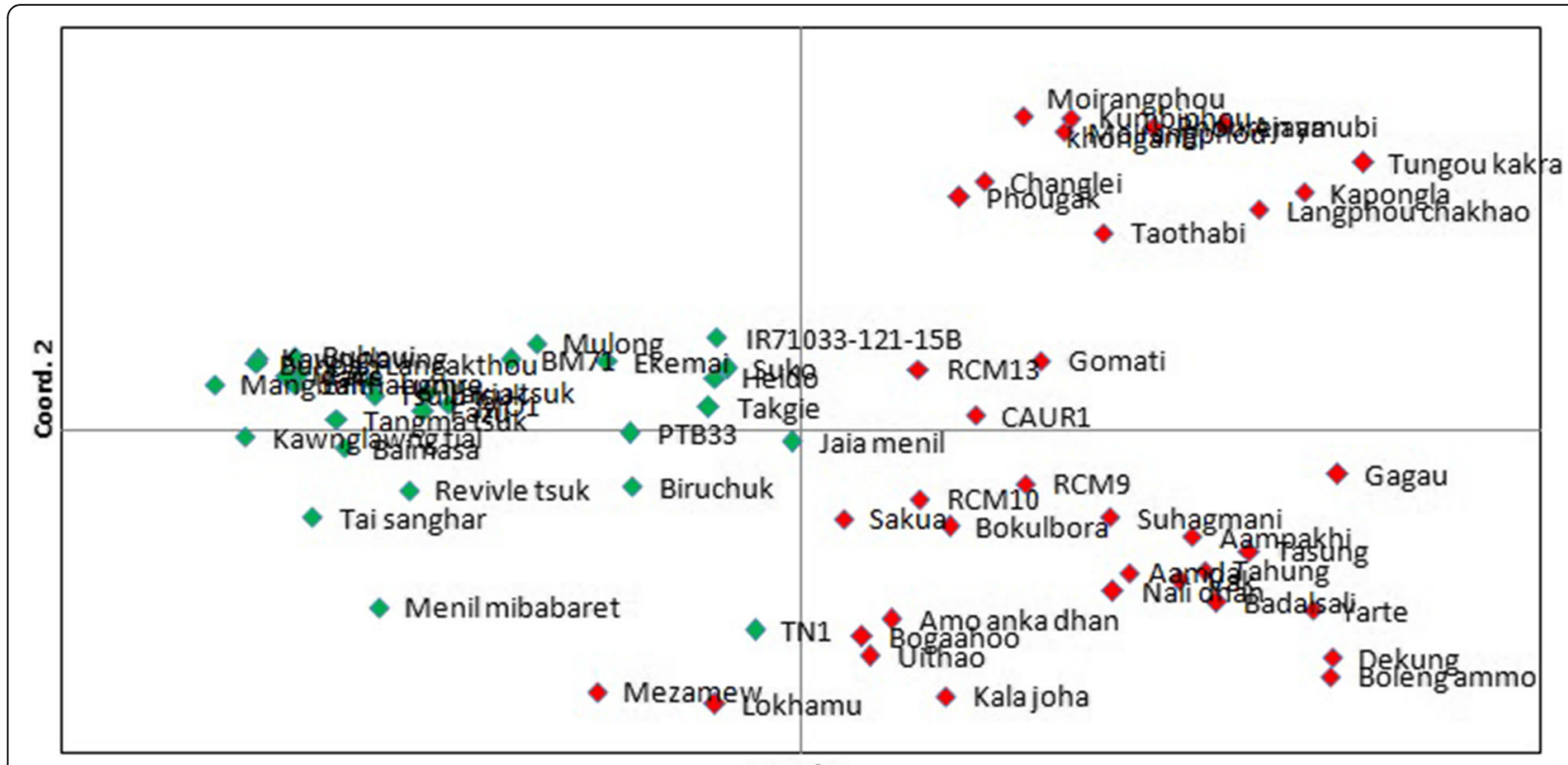

Coord. 1

Fig. 5 Principal coordinates analysis of 65 rice cultivars

needed to further find out higher contents of $\mathrm{Zn}$ and Fe since these micronutrients are essential for human health and development.

The NE rice cultivars contain considerable genetic diversity and variable traits which might be good sources for various improvement programs [20]. All SSR markers used in the present study were found to be polymorphic. A combination of random and trait-linked markers was utilized since Yadav et al. [34] reported trait-linked markers gave higher value of genetic diversity and Polymorphism Information Content (PIC) in some Indian rice germplasm than random markers, whereas several other workers have shown high genetic diversity in NE rice cultivars using random markers [20, $21,24]$. The number of alleles per locus (6.7727) found was higher than the ones reported earlier by Upadhyay et al. [35] (3.96 alleles per locus) and lower than that reported by Choudhury et al. [20] (13.57 alleles per locus). However, it was comparable with 7.9 alleles per locus reported by Das et al. [21]. The mean $\mathrm{H}_{\mathrm{E}}$ and PIC found in the present study showed a high value of heterozygosity index. The mean Fst values for all loci and between the two clusters were found to be 0.7786 and 0.1987 respectively indicating very high genetic differentiation among loci and among the clusters. Based on SSR analysis, there were seventeen highly informative markers (PIC> 0.50), viz., RM1, RM154, RM131, RM135, RM72, RM171, RM287, RM3825, RM246, RM260, RM525, RM219, RM223, RM8094, RM493, RM3412 and RM169; two informative markers (PIC between 0.25 and
0.50), RM153, RM125, and RM302; and two slightly informative markers $(\mathrm{PIC}<0.25)$, RM315 and RM443 $[36,37]$.

Population structure analysis using STRUCTURE showed highest $\Delta K$ value at $K=2$ revealing that the studied 65 rice cultivars were grouped into two clusters. The number of the cluster was in agreement to the previous studies: two clusters among 29 varieties of cultivated rice of NE India [20] and two clusters among 6 landraces of North-Western Indian Himalayas [38]. Roy et al. [24] have also reported a similar result of $K=2$, among hill rice of Arunachal Pradesh, NE India, belonging to indica and japonica. In the current study, the identified two main clusters can also be divided into sub-clusters corresponding to state-wise grouping. A similar result of state-wise grouping was also observed in aromatic rice germplasm from North Eastern India [13]. According to Evano et al. [39], alpha value closed to zero indicated that most of the individuals were from one population or another, and an alpha value greater than 1 indicated that most individuals were admixed. The observed small alpha value in this study (0.0663) might indicate that most of the individuals originated from one population or another.

In some areas of NE India, rice has been cultivated in shifting or jhum lands which only depend on the Monsoon rain. These cultivars survive in long spells of rainless weather and may be good candidates to look for these variable traits. Other important traits include dark color and aroma in Chakhao rice of Manipur, resistance against blast, 


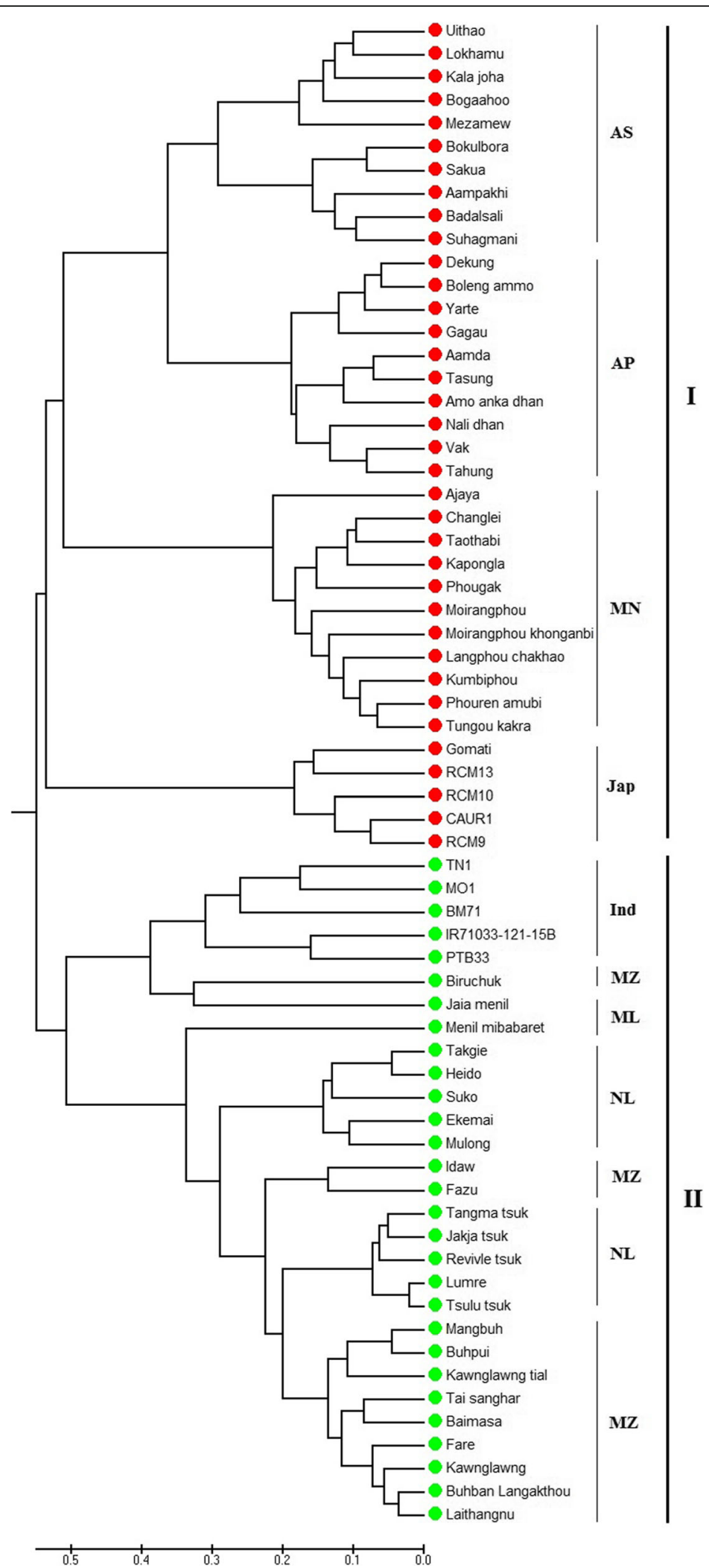

Fig. 6 UPGMA tree based on Nei's genetic distance (AS = Assam, AP = Arunachal Pradesh, MN = Manipur, MZ = Mizoram, ML = Meghalaya, $\mathrm{NL}=$ Nagaland, Ind = indica, Jap = japonica) 
Table 3 Correlations of genetic diversity, Zn and Fe content of 65 rice varieties, indicating no significance for all the test entries at $p<0.05$

\begin{tabular}{llll}
\hline & GD & Zn & Fe \\
\hline GD & 1 & & \\
Zn & $p=--$ & & \\
& 0.2265 & 1 & \\
Fe & $p=.070$ & $p=---$ & 1 \\
& -0.0478 & -0.032 & $p=--$ \\
\hline
\end{tabular}

GD Genetic diversity, Zn Zinc, Fe Iron

resistance to gall midge, deep water tolerance in Baon of Assam, drought resistance in Hmawrhang of Mizoram, etc. $[15,40,41]$. As evident from the current study, the genetic diversity of indigenous rice cultivars was found to be higher than that of agronomically improved varieties. These results are in agreement to a similar pattern observed for rice varieties of the Eastern Himalayan region of Northeast India [20]. The use of such genetic variability in breeding programs is a key factor for crop improvement [42]. Among the studied rice cultivars, Nalidhan cultivar of Arunachal Pradesh possessed the highest genetic diversity, followed by Vak and Boleng ammo cultivars. These high genetic diversity cultivars are promising candidates as sources for effective breeding or future rice improvement programs. However, some cultivars such as Moirangphou khonganbi, Moirangphou possessed a low level of genetic diversity suggesting necessary actionsshould be taken on the conservation of these landraces. Cultivars such as Vak, Bogaahoo and Tsulu tsuk possessed high genetic diversity and high $\mathrm{Zn}$ concentration. Similarly,

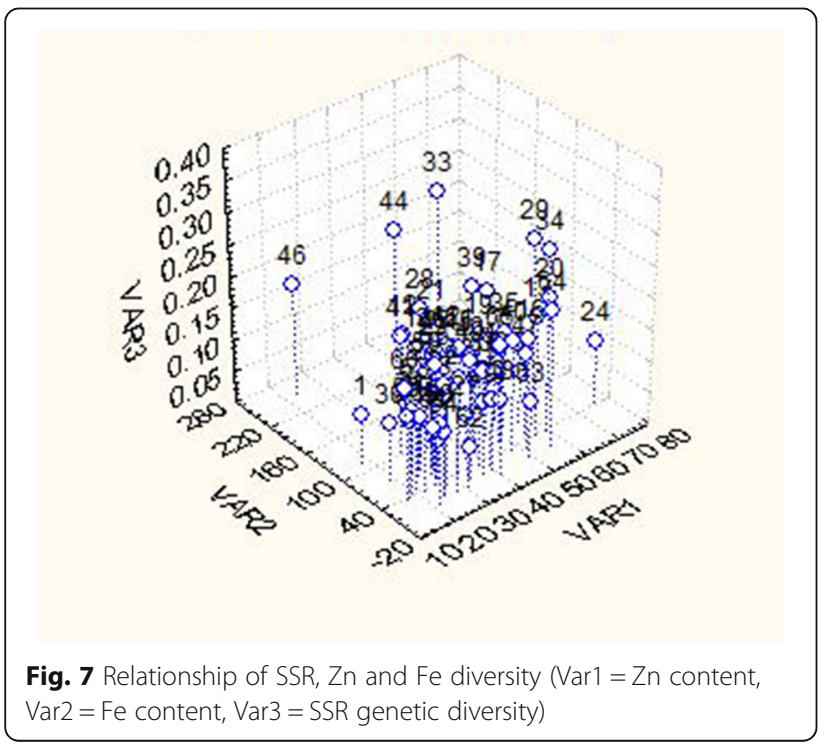

Kawnglawng, Jakjatsuk, Yarte, Mezamew, etc. possessed high $\mathrm{Fe}$ content and high genetic diversity. Nalidhan, the cultivar with the highest genetic diversity also possessed $\mathrm{Zn}$ and Fe contents higher than the average observed for these studied populations. Lumre also possessed high genetic diversity, high $\mathrm{Zn}$ and average Fe content. The highest $\mathrm{Zn}$ containing Badalsali cultivar possessed a lower genetic diversity than the average of all the studied populations. Similarly, the Fazu cultivar with the highest Fe content showed lower genetic diversity than the average of all the studied populations. The present investigation showed that the majority of the cultivars with high genetic diversity had high $\mathrm{Zn}$ contents and many cultivars also exhibited high genetic diversity along with high $\mathrm{Fe}$ content.

\section{Conclusion}

The current study provides a better understanding of genetic structure, diversity, and micronutrient $(\mathrm{Zn}$ and $\mathrm{Fe})$ richness in the indigenous rice cultivars of NE India. The cultivars possessing high genetic diversity (Nali dhan), high Zn (Badalsali) and Fe (Fazu) contents are promising candidates as parental lines for future rice breeding programs. These findings will further facilitate the conservation strategies and utilization of these landraces for developing sustainable rice improvement programs.

\section{Methods}

Plant material, collection and planting

Rice landraces were collected from six states of NE India. Details of collection sites are shown in Table 4. Indica and japonica check varieties were kind gifts from ICGEB, New Delhi, ABF, Hyderabad and ICAR, Kolasib. For isolation of DNA, individual cultivars were planted on polypots at Department of Botany, Mizoram University, India.

\section{Estimation of $\mathrm{Zn}$ and Fe content}

Dehusked rice seeds were crushed into a fine powder using mortar and pestle. The powdered sample $(0.1 \mathrm{~g})$ was placed in a $100 \mathrm{ml}$ conical flask and $20 \mathrm{ml}$ of Nitric acid $\left(\mathrm{HNO}_{3}\right)$ was added to it. The mixture was kept on a hot plate till the fuming of nitrogen dioxide ceased. Another $20 \mathrm{ml}$ of $\mathrm{HNO}_{3}$ was added and the samples were kept on the hot plate at a high temperature until the solution turned colourless. Then hydrogen peroxide $\left(\mathrm{H}_{2} \mathrm{O}_{2}\right)$ was added to make the solution colourless. The mixture was heated until the solution was reduced to 3$5 \mathrm{ml}$. This extract was diluted to $20 \mathrm{ml}$ with de-ionized water and then filtered through Whatman filter paper 1 . The extract was then injected into Atomic Absorption Spectrophotometer (Shimadzu AA-7000, Japan) and the results were expressed in $\mu \mathrm{g} / \mathrm{g}$. 
Table 4 Collected rice cultivars of NE India

\begin{tabular}{|c|c|c|c|c|}
\hline SI. No. & Cultivar name & Place of collection & States & Type \\
\hline 1. & Kapongla & Kakching & Manipur & Landrace \\
\hline 2. & Ajaya & Kakching & Manipur & Landrace \\
\hline 3. & Phougak & Kakching & Manipur & Landrace \\
\hline 4. & Phourenamubi & Thoubal & Manipur & Landrace \\
\hline 5. & Moirangphou & Thoubal & Manipur & Landrace \\
\hline 6. & Langphouchakhao & Kakching & Manipur & Landrace \\
\hline 7. & Changlei & Kakching & Manipur & Landrace \\
\hline 8. & Taothabi & Kakching & Manipur & Landrace \\
\hline 9. & Kumbiphou & Kakching & Manipur & Landrace \\
\hline 10. & Tungoukakra & Thoubal & Manipur & Landrace \\
\hline 11. & Moirangphoukhonganbi & Thoubal & Manipur & Landrace \\
\hline 12. & Takgie & Peren & Nagaland & Landrace \\
\hline 13. & Heido & Peren & Nagaland & Landrace \\
\hline 14. & Ekemai & Peren & Nagaland & Landrace \\
\hline 15. & Suko & Kohima & Nagaland & Landrace \\
\hline 16. & Mulong & Tuensang & Nagaland & Landrace \\
\hline 17. & Lumre & Tuensang & Nagaland & Landrace \\
\hline 18. & Tangmatsuk & Mokokchung & Nagaland & Landrace \\
\hline 19. & Revivletsuk & Mokokchung & Nagaland & Landrace \\
\hline 20. & Tsulu tsuk & Mokokchung & Nagaland & Landrace \\
\hline 21. & Jakjatsuk & Mokokchung & Nagaland & Landrace \\
\hline 22. & Mezamew & NC Hills & Assam & Landrace \\
\hline 23. & Uithao & NC Hills & Assam & Landrace \\
\hline 24. & Badalsali & Sonitpur & Assam & Landrace \\
\hline 25. & Bokulbora & Sonitpur & Assam & Landrace \\
\hline 26. & Kalajoha & Dheemaj & Assam & Landrace \\
\hline 27. & Sakua & Lakhimpur & Assam & Landrace \\
\hline 28. & Aampakhi & Lakhimpur & Assam & Landrace \\
\hline 29. & Bogaahoo & Dheemaj & Assam & Landrace \\
\hline 30. & Suhagmani & Dheemaj & Assam & Landrace \\
\hline 31. & Lokhamu & NC Hills & Assam & Landrace \\
\hline 32. & Aamda & West Siang & Arunachal Pradesh & Landrace \\
\hline 33. & Nalidhan & West Siang & Arunachal Pradesh & Landrace \\
\hline 34. & Vak & West Siang & Arunachal Pradesh & Landrace \\
\hline 35. & Amoankadhan & West Siang & Arunachal Pradesh & Landrace \\
\hline 36. & Tahung & East Siang & Arunachal Pradesh & Landrace \\
\hline 37. & Tasung & East Siang & Arunachal Pradesh & Landrace \\
\hline 38. & Dekung & West Siang & Arunachal Pradesh & Landrace \\
\hline 39. & Boleng ammo & East Siang & Arunachal Pradesh & Landrace \\
\hline 40. & Yarte & East Siang & Arunachal Pradesh & Landrace \\
\hline 41. & Gagau & East Siang & Arunachal Pradesh & Landrace \\
\hline 42. & Biruchuk & Lawngtlai & Mizoram & Landrace \\
\hline 43. & Kawnglawng & Diltlang South & Mizoram & Landrace \\
\hline 44. & Fare & Diltlang South & Mizoram & Landrace \\
\hline
\end{tabular}


Table 4 Collected rice cultivars of NE India (Continued)

\begin{tabular}{|c|c|c|c|c|}
\hline SI. No. & Cultivar name & Place of collection & States & Type \\
\hline 45. & Kawnglawngtial & Mualbukawnpui & Mizoram & $\overline{\text { Landrace }}$ \\
\hline 46. & BuhbanLangakthou & Vawmbuk & Mizoram & Landrace \\
\hline 47. & Laithangnu & Darlawn & Mizoram & Landrace \\
\hline 48. & Tai sanghar & Darlawn & Mizoram & Landrace \\
\hline 49. & Baimasa & Phuaibuang & Mizoram & Landrace \\
\hline 50. & Idaw & Tlungvel & Mizoram & Landrace \\
\hline 51. & Mangbuh & Chhingchhip & Mizoram & Landrace \\
\hline 52. & Buhpui & N Chaltlang & Mizoram & Landrace \\
\hline 53. & Fazu & Saichal & Mizoram & Landrace \\
\hline 54. & Jaiamenil & East Garo Hills & Meghalaya & Landrace \\
\hline 55. & Menilmibabaret & East Garo Hills & Meghalaya & Landrace \\
\hline 56. & $\mathrm{TN} 1^{\mathrm{a}}$ & ICGEB & New Delhi & Improved \\
\hline 57. & $\mathrm{BM} 71^{\mathrm{a}}$ & ABF, Hyderabad & Telangana & Improved \\
\hline 58. & IR71033-121-15B ${ }^{a}$ & ABF, Hyderabad & Telangana & Improved \\
\hline 59. & $\mathrm{MO}^{\mathrm{a}}$ & ABF, Hyderabad & Telangana & Improved \\
\hline 60. & PTB33 ${ }^{a}$ & ABF, Hyderabad & Telangana & Improved \\
\hline 61. & CAUR1 ${ }^{b}$ & ICAR, Kolasib & Mizoram & Improved \\
\hline 62. & Gomati $^{\text {b }}$ & ICAR, Kolasib & Mizoram & Improved \\
\hline 63. & RCM9 ${ }^{b}$ & ICAR, Kolasib & Mizoram & Improved \\
\hline 64. & $\mathrm{RCM} 10^{\mathrm{b}}$ & ICAR, Kolasib & Mizoram & Improved \\
\hline 65. & RCM13 b & ICAR, Kolasib & Mizoram & Improved \\
\hline
\end{tabular}

${ }^{a}$ representsIndica varieties, ${ }^{b}$ representsJaponica varieties. ABF Agri Biotech Foundation, ICGEB International Centre for Genetic Engineering and Biotechnology, ICAR Indian Council of Agricultural Research

\section{Genomic DNA isolation and PCR amplification}

Genomic DNA was isolated from 15-day old seedlings following Edwards et al. [43]. Single leaflet of 15-day old seedling was used for isolation of DNA. The leaflet was macerated using a micropestle in a $1.5 \mathrm{ml}$ centrifuge tube. After maceration, $400 \mu \mathrm{l}$ of extraction buffer (200 $\mathrm{mM}$ Tris $\mathrm{HCl} \mathrm{pH} 7.5,250 \mathrm{mMNaCl}, 25 \mathrm{mM}$ EDTA, $0.5 \%$ SDS) was added to the tube. The sample was then vortexed vigorously for $1 \mathrm{~min}$ and centrifuged at 13000 $\mathrm{rpm}$ for $5 \mathrm{~min}$. Then, $300 \mu \mathrm{l}$ of the supernatant was transferred to a fresh centrifuge tube and an equal volume of Isopropanol was added. The samples were kept at room temperature for $2 \mathrm{~min}$ and then were centrifuged at $13,000 \mathrm{rpm}$ for $5 \mathrm{~min}$. The resulting pellets were air dried at room temperature and dissolved in $100 \mu \mathrm{l} \mathrm{TE}(10 \mathrm{mM}$ Tris, $1 \mathrm{mM}$ EDTA) buffer.

Twenty-two simple sequence repeats (SSR) primers (Table 5) were used for amplification of genomic DNA. Amplification was performed in ABI Veriti 96 well Thermal cycler (ABI, USA) in $25 \mu \mathrm{l}$ reaction containing 1X PCR buffer, $100 \mu \mathrm{M}$ dNTP mixture, $3 \mathrm{mM}$ $\mathrm{MgCl}_{2}, 1 \mathrm{U}$ Taq polymerase (Genie, India), $50 \mathrm{ng}$ of each primer and $50 \mathrm{ng}$ template DNA. The amplification conditions were set as, initial denaturation at $94{ }^{\circ} \mathrm{C}$ for $5 \mathrm{~min}, 35$ cycles of denaturation at $94{ }^{\circ} \mathrm{C}$ for $30 \mathrm{~s}$, annealing for $30 \mathrm{~s}$, extension at $72^{\circ} \mathrm{C}$ for $1 \mathrm{~min}$ followed by a final extension at $72{ }^{\circ} \mathrm{C}$ for $7 \mathrm{~min}$. The amplified products were electrophoresed on 2.5\% agarose gel and visualized by standard ethidium bromide staining [43, 44].

\section{Genetic data analysis}

Bands were scored using Alpha View software (Alpha Imager, Protein Simple, USA). Total number of alleles, number of effective alleles, number of polymorphic loci, observed and expected heterozygosity, Nei'sgenetic diversity [45], Fst, and population-wise diversity were calculated using genetic analysis package POPGENE 1.31 [46]. Major allele frequency (MAF) and the polymorphism information content (PIC) were calculated using PowerMarker 3.25 [47]. Analysis of molecular variance (AMOVA) and principal co-ordinates analysis (PCoA) were performed in GenAlEx 6.5 [48]. The unweighted pair group method with an arithmetic 
Table 5 Details of SSR primers used (http://gramene.org/markers/microsat/all-ssr.html)

\begin{tabular}{|c|c|c|c|c|c|c|}
\hline $\mathrm{SINO}$ & Primer name & Sequences (Forward primer/Reverse primer) & Chr.no. & Marker selection & $\mathrm{T}_{\mathrm{a}}\left({ }^{\circ} \mathrm{C}\right)$ & Expected amplicon size (bp) \\
\hline 1. & RM1 & $\begin{array}{l}\text { Fp - 5'-GCGAAAACACAATGCAAAAA-3' } \\
\text { Rp - 5'-GCGTTGGTGGACCTGAC-3' }\end{array}$ & 1 & Random & 55 & 113 \\
\hline 2. & RM154 & $\begin{array}{l}\text { Fp - 5'-ACCCTCTCCGCCTCGCCTCCTC-3' } \\
\text { Rp - 5'-CTCCTCCTCCTGCGACCGCTCC-3' }\end{array}$ & 2 & Random & 61 & 183 \\
\hline 3. & RM131 & $\begin{array}{l}\text { Fp - 5'-TCCTCCCTCCCTTCGCCCACTG-3' } \\
\text { Rp - 5'-CGATGTTCGCCATGGCGTCTCC-3' }\end{array}$ & 4 & Random & 61 & 215 \\
\hline 4. & RM135 & $\begin{array}{l}\text { Fp - 5'-CTCTGTCTCCTCCCCCGCGTCG-3' } \\
\text { Rp - 5'-TCAGCTTCTGGCCGGCCTCCTC-3' }\end{array}$ & 3 & Random & 55 & 131 \\
\hline 5. & RM153 & $\begin{array}{l}\text { Fp - 5'-GCCTCGAGCATCATCATCAG-3' } \\
\text { Rp - 5'-ATCAACCTGCACTTGCCTGG-3' }\end{array}$ & 5 & Random & 55 & 201 \\
\hline 6. & RM125 & $\begin{array}{l}\text { Fp - 5'-ATCAGCAGCCATGGCAGCGACC-3' } \\
\text { Rp - 5'-AGGGGATCATGTGCCGAAGGCC-3' }\end{array}$ & 7 & Random & 55 & 127 \\
\hline 7. & RM72 & $\begin{array}{l}\text { Fp - 5'-CCGGCGATAAAACAATGAG-3' } \\
\text { Rp - 5'-GCATCGGTACTAACTAAGGG-3' }\end{array}$ & 8 & Random & 55 & 166 \\
\hline 8. & RM171 & $\begin{array}{l}\text { Fp - 5'-CGATCCATTCCTGCTGCTCGCG-3' } \\
\text { Rp - 5'-CGCCCCCATGCATGAGAAGACG-3' }\end{array}$ & 10 & Random & 55 & 328 \\
\hline 9. & RM287 & $\begin{array}{l}\text { Fp - 5'-TTCCCTGTTAAGAGAGAAATC-3' } \\
\text { Rp - 5'-GTGTATTGGTGAAAGCAAC-3' }\end{array}$ & 11 & Random & 55 & 118 \\
\hline 10. & RM302 & $\begin{array}{l}\text { Fp - 5'-TCATGTCATCTACCATCACAC-3' } \\
\text { Rp - 5'-ATGGAGAAGATGGAATACTTGC-3' }\end{array}$ & 1 & Trait-linked (drought) & 55 & 156 \\
\hline 11. & RM3825 & $\begin{array}{l}\text { Fp - 5'-AAAGCCCCCAAAAGCAGTAC-3' } \\
\text { Rp - 5'-GAGCTCCATCAGCCATTCAG-3' }\end{array}$ & 1 & Trait-linked (drought) & 55 & 147 \\
\hline 12. & RM246 & $\begin{array}{l}\text { Fp - 5'-GAGCTCCATCAGCCATTCAG-3' } \\
\text { Rp - 5'-CTGAGTGCTGCTGCGACT-3' }\end{array}$ & 1 & Trait-linked (drought) & 55 & 116 \\
\hline 13. & RM260 & $\begin{array}{l}\text { Fp - 5'-ACTCCACTATGACCCAGAG-3' } \\
\text { Rp - 5'-GAACAATCCCTTCTACGATCG-3' }\end{array}$ & 12 & Trait-linked (drought) & 55 & 111 \\
\hline 14. & RM525 & $\begin{array}{l}\text { Fp - 5'-GGCCCGTCCAAGAAATATTG-3' } \\
\text { Rp - 5'-CGGTGAGACAGAATCCTTACG-3' }\end{array}$ & 2 & Trait-linked (drought) & 55 & 131 \\
\hline 15. & RM219 & $\begin{array}{l}\text { Fp - 5'-CGTCGGATGATGTAAAGCCT-3' } \\
\text { Rp - 5'-CATATCGGCATTCGCCTG-3' }\end{array}$ & 9 & Trait-linked (drought) & 55 & 202 \\
\hline 16. & RM315 & $\begin{array}{l}\text { Fp - 5'-GAGGTACTTCCTCCGTTCAC-3' } \\
\text { Rp - 5'-AGTCAGCTCACTGTGCAGTG-3' }\end{array}$ & 1 & Trait-linked (salt) & 55 & 133 \\
\hline 17. & RM223 & $\begin{array}{l}\text { Fp - 5'-GAGTGAGCTGGTGCTGAAAC-3' } \\
\text { Rp - 5'-GAAAGGCAAGTCTTGGCACTG-3' }\end{array}$ & 8 & Trait-linked (salt) & 55 & 165 \\
\hline 18. & RM8094 & $\begin{array}{l}\text { Fp - 5'-AAGTTTGTACACATCGTATACA-3' } \\
\text { Rp - 5'-CGCGACCAGTACTACTACTA-3' }\end{array}$ & 1 & Trait-linked (salt) & 55 & 209 \\
\hline 19. & RM493 & $\begin{array}{l}\text { Fp - 5'-TAGCTCCAACAGGATCGACC-3' } \\
\text { Rp - 5'-GTACGTAAACGCGGAAGGTG-3' }\end{array}$ & 1 & Trait-linked (salt) & 55 & 211 \\
\hline 20. & RM3412 & $\begin{array}{l}\text { Fp - 5'-AAAGCAGGTTITCCTCCTCC-3' } \\
\text { Rp - 5'-CCCATGTGCAATGTGTCTTC-3' }\end{array}$ & 1 & Trait-linked (salt) & 55 & 211 \\
\hline 21. & RM443 & $\begin{array}{l}\text { Fp - 5'-GATGGTTTCATCGGCTACG-3' } \\
\text { Rp - 5'-AGTCCCAGAATGTCGTTTCG-3' }\end{array}$ & 1 & Trait-linked (salt) & 55 & 124 \\
\hline 22. & RM169 & $\begin{array}{l}\text { Fp - 5'-TGGCTGGCTCCGTGGGTAGCTG-3' } \\
\text { Rp - 5'-TCCCGTTGCCGTTCATCCCTC-3' }\end{array}$ & 5 & Trait-linked (salt) & 67 & 167 \\
\hline
\end{tabular}

Chr. no. Chromosome number, $T_{a}$ Annealing temperature

mean (UPGMA) dendrogram was constructed using MEGA 6 [49] based on Nei's genetic distance. The possible population structure was analyzed using STRUCT URE 2.3.4 [50]. The parameter was set as 100,000 for the length of burn-in period and Markov Chain Monte Carlo (MCMC) repeats after burn-in was set as 100,
000. A possible number of subpopulations (K) was set from $K=1$ to $K=10$. Structure Harvester [51] was used to find the final $\mathrm{K}$ value. Then, the relationship among genetic diversity (gene diversity), $\mathrm{Zn}$ and $\mathrm{Fe}$ contents were measured using STATISTICA 5.0 (Statsoft Inc., USA, 1995). 


\section{Supplementary information}

Supplementary information accompanies this paper at https://doi.org/10. 1186/s12863-019-0780-6.

Additional file 1. The genotype data of indigenous rice cultivars of NE India and improved varieties.

Additional file 2. Pairwise Population Matrix of Nei Genetic Identity.

Additional file 3. Pairwise Population Fst Values.

Additional file 4. Summary of analysis of molecular variance.

\section{Abbreaviations}

AMOVA: Analysis of molecular variance; Fst: Genetic differentiation; $\mathrm{H}_{\mathrm{E}}$ : Expected heterozygosity; HYV: High yielding variety; MAF: Major allele frequency; MCMC: Markov Chain Monte Carlo; na: Number of alleles; ne: Effective number of alleles; Nei: Nei's genetic distance; PCoA: Principal coordinates analysis; PIC: Polymorphism information content; UPGMA: The unweighted pair group method with an arithmetic mean

\section{Acknowledgments}

Authors thank NBPGR, Umium, Meghalaya for the kind supply of rice cultivars. Authors are thankful to Dr. Usha Bhardwaj for her constructive comments and thoughtful ideas. Authors also thank all the farmers for their kind gift.

\section{Authors' contributions}

VS and SPS performed the experiments and analyzed the data. YTS conceived, designed the experiments and analyzed the data. All authors have read and approved the final manuscript.

\section{Funding}

Science and Engineering Research Board, Govt. of India (SB/FT/LS-381/2012) and University Grants Commission, Govt. of India (F. 20 (26)/2012 (BSR)) are acknowledged for financial supports. The funders had no role in the design of the study, collection, analysis, or interpretation of the data and in writing the manuscript.

\section{Availability of data and materials}

All data supporting the conclusions of this article are included within the article and its Additional files 1, 2,3 and 4

\section{Ethics approval and consent to participate}

The authors declare that no permission was required for sampling.

\section{Consent for publication}

Not applicable.

\section{Competing interests}

The authors declare that they have no competing interests.

Received: 30 May 2019 Accepted: 21 September 2019

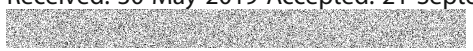

\section{References}

1. Bandumula N. Rice Production in Asia: Key to Global Food Security. Proc Natl Acad Sci India Sect B Biol Sci. 2018;88:1323.

2. Bouis H, Welch R. Biofortification — a sustainable agricultural strategy for reducing micronutrient malnutrition in the global south. Crop Sci. 2010;50:20-32.

3. Kennedy G, Nantel G, Shetty P. The scourge of "hidden hunger": global dimensions of micronnutrientdefi ciencies. Food Nutr Agric. 2003;32:8-16.

4. Hambridge M. Human zinc deficiency. J Nutr. 2000;130(5):1344S-9S.

5. Zhang C. Essential functions of iron-requiring proteins in DNA replication, repair and cell cycle control. Plant Cell. 2014;5(10):750-60.

6. Roohani N, Hurrell R, Kelishadi R, Schulin R. Zinc and its importance for human health: an integrative review. J Res Med Sci. 2013;18(2):144-57.

7. Prasad AS. Discovery of human zinc deficiency: its impact on human health and disease. Adv Nutr. 2013;4(2):176-90.

8. Ehrlich PR, Ehrlich AH, Daily GC. Food security, population and environment. Popul Dev Rev. 1993;19(1):1-32.

9. Goddard AF, James MW, McIntyre AS, Scott BB. Guidelines for the management of iron deficiency anaemia. Gut. 2011;60(10):1309-16.
10. De Falco L, Sanchez M, Silvestri L, Kannengiesser C, Muckenthaler MU, Iolascon A, Gouya L, Camaschella C, Beaumont C. Iron refractory iron deficiency anemia. Haematologica. 2013;98:845-53.

11. Miller JL. Iron deficiency anemia: a common and curable disease. Cold Spring HarbPerspect Med. 2013;3(7):a011866.

12. Brown $\mathrm{KH}$, Wuehler SE, Peerson JM. The importance of zinc in human nutrition and estimation of the global prevalence of zinc deficiency. Food Nutr Bull. 2001;22(2):113-25.

13. Lee Al, Okam MM. Anemia in Pregnancy. Hematol Oncol Clin N Am. 2011; 25:241-59.

14. Myers N. Threatened biotas: "hots spots" in tropical forests. Environmentalist 1988;8(3):187-208

15. Hore DK. Rice diversity collection, conservation and management in northeastern India. Genet Resour Crop Evol. 2005;52:1129-40

16. Roy S, Banerjee A, Mawkhlieng B, Misra AK, Pattanayak A, Harish GD, Singh SK, Ngachan SV, Bansal KC. Genetic diversity and population structure in aromatic and quality rice (Oryza sativa L.) landraces from north-eastern India. PLoS One. 2015;10(6):e0129607.

17. Enjuanes C, Klip IT, Bruguera J, Cladellas M, Ponikowski P, Banasiak W, van Veldhuisen DJ, van der Meer P, Jankowska EA, Comin-Colet J. Iron deficiency and health-related quality of life in chronic heart failure: results from a multicenter European study. Int J Cardiol. 2014;174:268-75.

18. Gupta PK, Varshney RK. The development and use of microsatellite markers for genetic analysis and plant breeding with emphasis on bread wheet. Euphytica. 2000;113:163-85.

19. Li FP, Lee YS, Kwon SW, Li G, Park YJ. Analysis of genetic diversity and trait correlations among Korean landrace rice (Oryza sativa L.). Genet Mol Res. 2014;13(3):6316-31.

20. Choudhury B, Khan ML, Dayanandan S. Genetic structure and diversity of indigenous rice (Oryza sativa) varieties in the eastern Himalayan region of Northeast India. SpringerPlus. 2013;2:228.

21. Das B, Sengupta S, Parida SK, Roy B, Ghosh M, Prasad M, Ghose TK. Genetic diversity and population structure of rice landraces from eastern and north eastern states of India. BMC Genet. 2013;14:71.

22. Choudhury DR, Singh N, Singh AK, Kumar S, Srinivasan K, Tyagi RK, Ahmad A, Singh NK, Singh R. Analysis of genetic diversity and population structure of rice germplasm from north-eastern region of India and development of a core germplasm set. PLoS One. 2014;9(11):e113094.

23. Roy SC, Sharma BD. Assessment of genetic diversity in rice (Oryza sativa L.) germplasm based on agro-morphology traits and zinc-iron content for crop improvement. PhysiolMolBiol Plants. 2014;20(2):209-24.

24. Roy S, Marndi BC, Mawkhlieng B, Banerjee A, Yadav RM, Misra AK, Bansal KC. Genetic diversity and structure in hill rice (Oryza sativa L.) landraces from the north-eastern Himalayas of India. BMC Genet. 2016;17:107.

25. Vanlalsanga, Singh YT. Genetic diversity and population structure in upland rice (Oryza sativa L.) of Mizoram, north East India as revealed by morphological, biochemical and molecular markers. Biochem Genet. 2019:57:421-42.

26. Meng F, Wei $Y$, Yang X. Iron content and bioavailability in rice. J Trace Elem Med Biol. 2005;18(4):333-8.

27. Scheider JM, Fujii ML, Lamp CL, Lonnerdal B, Dewey KG, Zidenberg-Cher S Anemia, iron deficiency, and iron deficiency anemia in 12-36-mo-old children from low-income families. Am J ClinNutr. 2005;82:1269-75.

28. Anuradha K, Agarwal S, Batchu AK, Babu AP, Swamy BPM, Longvah T, Sarla $\mathrm{N}$. Evaluating rice germplasm for iron and zinc concentration in brown rice and seed dimensions. J Phytol. 2012:4(1):19-25.

29. Narayanan NN, Vasconcelos MW, Grusak MA. Expression profiling of Oryza sativa metal homeostasis genes in different rice cultivars using a cDNA macroarray. Plant Physiol Biochem. 2007;45:277-86.

30. Brar B, Jain S, Singh R, Jain RK. Genetic diversity for iron and zinc contents in a collection of 220 rice ( Onzza sativa L.) genotypes. Indian J. Genet. 2011;71(1):67-73.

31. Dikshit N, Sivaraj N, Sultan SM, Datta M. Diversity analysis of grain characteristics and micronutrient content in rice landraces of Tripura, India. Bangladesh J Bot. 2016;45(5):1143-9.

32. Jajda HM, Patel KG, Patel SR, Solanki VH, Patel KN, Singhh S. Comparative efficacy of two standard methods for determination of iron and zinc in fruits, pulses and cereals. J Food Sci Technol. 2015:52(2):1096-102.

33. Verma DK, Srivastav PP. Proximate composition, mineral content and fatty acids analyses of aromatic and non-aromatic Indian rice. Rice Sci. 2017;24(1):21-31.

34. Yadav S, Singh A, Singh MR, Goel N, Vinod KK, Mohapatra T, Singh AK. Assessment of genetic diversity in Indian rice germplasm (Oryza sativa L.): use of random versus trait-linked microsatellite markers. J Genet. 2013;92:545-57. 
35. Upadhyay P, Neeraja CN, Kole C, Singh VK. Population structure and genetic diversity in popular rice varieties of India as evidenced from SSR analysis. Biochem Genet. 2012;50:770-83.

36. Botstein D, White RL, Skolnick M, Davis RW. Construction ofa genetic linkage map in man using restriction fragment lengthpolymorphism. Am J Hum Genet. 1980;32:314-31.

37. Freeg HA, Anis GB, Abo-Shousha AA, El-Banna AN, El-Sabagh A. Genetic diversity among some rice genotypes with different drought tolerance based on SSR markers. CercetAgron Mold. 2016;3(169):39-50.

38. Gayacharan, Bisht IS, Pandey A, Yadav MC, Rana JC. Population structure of upland red rice (Oryza sativa L.) landraces from north-western Indian Himalayas. Ind J Biotechnol. 2015;14:42-8.

39. Evanno G, Regnaut $\mathrm{S}$, Goudet J. Detecting the number of clusters of individuals using the software structure: a simulation study. Mol Ecol. 2005; 14(8):2611-20.

40. Singh Kl, Singh MP, Sharma PR, Singh TR. Screening of certain promising local rice varieties for resistance to the Manipur gall midge (Orseoliaoryzae wood-Mason) biotype. Indian J Entomol. 2006;68(3):265-8.

41. Mahender A, Swain DM, Gitishree D, Subudhi HN, Rao GJN. Molecular analysis of native Manipur rice accessions for resistance against blast. Afr J Biotechnol. 2012;11:1321-9.

42. Babu BK, Meena V, Agarwal V, Agarwal PK. Population structure and genetic diversity analysis of Indian and exotic rice (Oryza sativa L.) accessions using SSR markers. Mol Biol Rep. 2014. https://doi.org/10.1007/s11033-014-3304-5.

43. Edwards K, Johnstone C, Thomson C. A simple and rapid method for the preparation of plant genomic DNA for PCR analysis. Nucleic Acids Res. 1991; 19(6):1349.

44. Sambrook J, MacCallum P, Russell D. Molecular cloning: a laboratory manual 3. Cold Spring Harbor: Cold Spring Harbor Laboratory Press; 2001.

45. Nei M. Analysis of gene diversity in subdivided populations. Proc Natl Acad Sci U S A. 1973;70:3321-3.

46. Yeh FC, Yang R, Boyle T. POPGENE version 1.32. Ag/for molecular biology and biotechnology Centre, University of Alberta and Center for International Forestry Research; 1999.

47. Liu K, Muse SV. PowerMarker: integrated analysis environment for genetic marker data. Bioinformatics. 2005;21(9):2128-9.

48. Peakall R, Smouse PE. GenAIEx 6.5: genetic analysis in excel. Population genetic software for teaching and research - an update. Bioinformatics. 2012;28(19):2537-9.

49. Tamura K, Stecher G, Peterson D, Filipski A, Kumar S. MEGA6: molecular evolutionary genetics analysis version 6.0. MolBiolEvol. 2013;30:2725-9.

50. Pritchard JK, Stephens M, Donnelly P. Inference of population structure using multilocus genotype data. Genetics. 2000;155:945-59.

51. Earl DA, von Holdt BM. Structure harvester: a website and program for visualizing structure output and implementing the Evanno method. Conser Genet Resour. 2012;4(2):359-61.

\section{Publisher's Note}

Springer Nature remains neutral with regard to jurisdictional claims in published maps and institutional affiliations.

Ready to submit your research? Choose BMC and benefit from:

- fast, convenient online submission

- thorough peer review by experienced researchers in your field

- rapid publication on acceptance

- support for research data, including large and complex data types

- gold Open Access which fosters wider collaboration and increased citations

- maximum visibility for your research: over $100 \mathrm{M}$ website views per year

At $\mathrm{BMC}$, research is always in progress.

Learn more biomedcentral.com/submissions 